\title{
THE LUMINOSITY FUNCTION OF WHITE DWARFS IN THE LOCAL DISK AND HALO
}

\author{
James Liebert \\ Steward Observatory, University of Arizona \\ Tucson, Arizona 85721 \\ Conard C. Dahn and David G. Monet \\ U. S. Naval Observatory \\ Flagstaff, Arizona 86002
}

\section{INTRODUCTION}

The luminosity function (LF) and total space density of white dwarfs in the solar neighborhood contain important information about the star formation history of the stellar population, and provide an independent method of measuring its age. The first empirical estimates of the LF for degenerate stars were those of Weidemann (1967), Kovetz and Shaviv (1976) and Sion and Liebert (1977). The follow-up investigations made possible by the huge Luyten Palomar proper motion surveys, however, added many more faint white dwarfs to the known sample. While the number of known cool white dwarfs grew to nearly one hundred, these did not include any that were much fainter intrinsically than the coolest degenerates found from the early Luyten, van Biesbroeck and Eggen-Greenstein lists.

In an analysis of a fainter sample, Liebert et al. (1979) first discussed the evidence that there was a paucity of white dwarfs fainter than $M_{\mathbf{v}} \sim+16$ with respect to the predictions of theoretical cooling models allowing cooling ages greater than 10 Gyrs. Taken at face value, the result implied that the bulk of star formation in the disk began less than 10 Gyrs ago, an effective age for the disk billions of years younger than the ages estimated for globular clusters and the galactic halo (Liebert 1980). These results also implied that degenerate dwarfs are a relatively minor contributor to the local mass density of the galactic disk.

The last several years have brought considerable improvements in both the theoretical calculations and the observational data base. Iben and Tutukov (1984) presented new evolutionary models and rediscussed the published observational data, offering alternative explanations for an apparent paucity of very faint degenerate stars. Winget et al. (1987) combined a new set of theoretical calculations with a preliminary version of the observational data base reported here and reported a direct estimate of the age of the galactic disk of $9.3 \pm 2$ billion years. However, Winget 
and Van Horn (1987) discussed in considerable detail the sensitivity of this number - which basically represents the cooling time of a model to reach a luminosity near $\log \left(\mathrm{L} / \mathrm{L}_{\odot}\right) \sim-4.5-$ to various physical parameters in the models. The use of different parameters explains the discrepancies between their conclusion and the result of D'Antona and Mazzitelli (1986).

The improved observational data base is documented in detail in (Liebert, Dahn and Monet 1988; hereafter LDM). While this paper includes a brief review of that work, we now can incorporate new parallax data and considerably improved absolute magnitude estimates for several stars in the sample. Thus Figure 1 is an updated observational LF, which supersedes that appearing in LDM. After the brief review and presentation of updated results in Section II, a discussion of the sources of error and incompleteness is given in Section III. The kinematics of the sample based on the derived tangential velocities is also discussed. Section IV features a preliminary look at the LF yielded by the sparse population of local halo white dwarfs, as defined from a kinematic criterion.

\section{THE LHS 8TENTHS SAMPLE}

The sample used in LDM consists of the 43 spectroscopically confirmed white dwarfs contained in the LHS Catalogue (Luyten 1979) which have (1) proper motions $(\mu) \geq 0$ !'800 $\mathrm{yr}^{-1}$, (2) locations in the sky north of $\delta=-20^{\circ}$, and (3) $\mathrm{M}_{\mathrm{v}} \geq 13.0$. Available astrometric, photometric and spectroscopic data for each star in the sample are summarized in Table 1 of LDM and the notes. Absolute magnitudes were calculated for each star, usually based on an available trigonometric parallax. Space densities were calculated using the $1 / \mathrm{V}_{\max }$ method similar to the application of Schmidt (1975). In this presentation, new or updated trigonometric parallaxes are substituted for six stars. These stars, with the improved absolute parallax values and mean errors in parentheses,

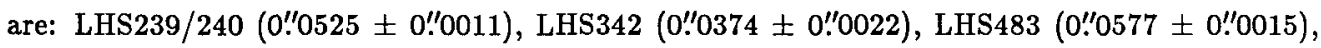
LHS542 (0.'0322 $\pm 0 . \prime 0037)$, LHS2364 (0."0416 $\left.\pm 0 .{ }^{\prime \prime} 0022\right)$ and LHS2673 (0."0284 $\left.\pm 0 . \prime 0033\right)$.

For a dwindling minority without trigonometric values, photometric parallaxes were assigned based on new determinations of the relationships between absolute visual magnitude, effective temperatures and broad band BVI colors. Particular attention was given to estimating the bolometric correction (BC), necessary to place the stars in bolometric luminosity intervals for comparison with theoretical predictions.

For the hotter DA stars, the available tabulations of BCs for hydrogen-rich atmosphere calculations show considerable scatter about what is expected to be a monotonic functional dependence on the effective temperature ( $\left.T_{\text {eff }}\right)$. Matt Wood has kindly pointed out to us that the mean $M_{b o l}$ bin centers for the hot white dwarfs listed in Table 4 of LDM differ slightly (i.e., up to approximately a symbol diameter) from the corresponding points plotted in Figures 3 and 4 of that

paper. This discrepancy resulted from inadvertently using preliminary values for the mean $\mathrm{M}_{\text {bol }}$ numbers in the figures rather than those finally adopted and presented in Table 4 of LDM. These preliminary values were based on slightly different smoothings of the model $\mathrm{BC}$ values used in estimating a functional relationship between $\mathrm{BC}$ and $\mathrm{T}_{\text {eff }}$ from that finally adopted for hot DA 
white dwarfs. Figure 1 includes the hot white dwarf LF from the Palomar Green Survey now plotted with a luminosity scale entirely consistent with Table 4 of LDM.

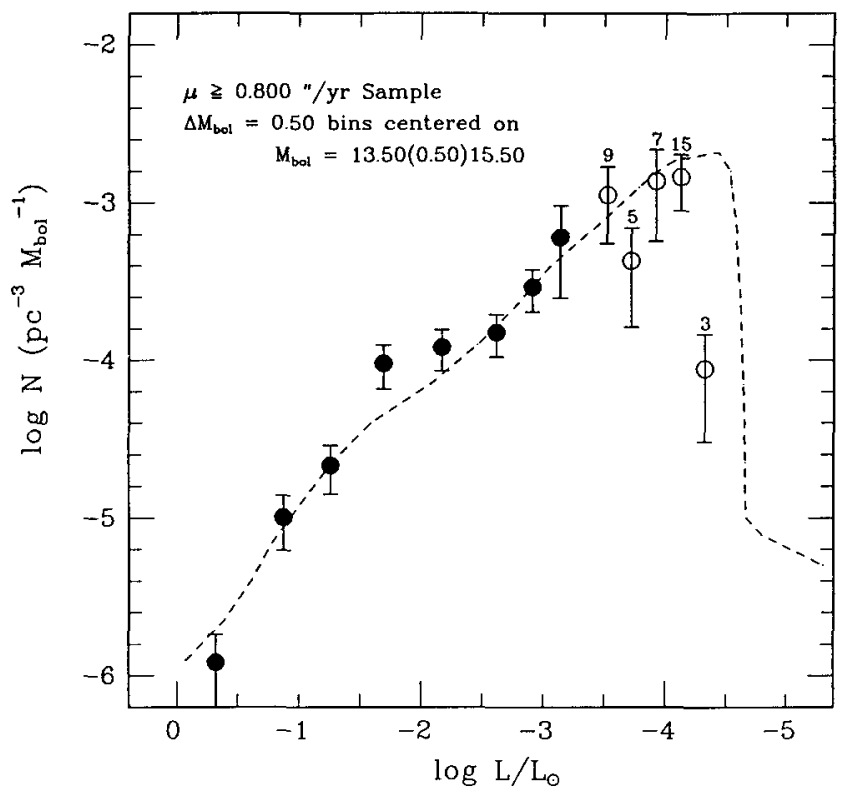

Figure 1. Luminosity function for disk white dwarfs, using blackbody BCs and updated from Fig. 3c of LDM by incorporating new trigonometric parallaxes. Numbers of WDs in each bin are listed above the tops of the bins.

For the cooler white dwarfs, the problem is that BCs for the more accurate calculations of the energy distributions of stars with helium-rich atmospheres are not generally available. In one approach, BCs were estimated by applying the value for the blackbody corresponding to tha derived $\mathrm{T}_{\text {eff }}$ consistent with the measured value of $\mathrm{M}_{\mathbf{v}}$. Arguments were given that the blackbody $\mathrm{BCs}$ probably overestimate the corrections for helium atmosphere white dwarfs. For several wellstudied cases, these imply larger radii and smaller masses than the means for DA white dwarfs. It is implausible that the older white dwarfs should have a smaller mean mass than that for the hotter DA stars. On the other hand, a conservative lower limit to the luminosities of the coolest stars in the sample (and an upper limit to the derived cooling ages for a given set of models) is provided by assuming no BCs at all! This was the alternative approach, and it was argued that the LFs presented from both methods bound the uncertainty due to the BCs.

\section{THE DISK WHITE DWARF LUMINOSITY FUNCTION: SOURCES OF ERROR AND INCOMPLETENESS}

While the observed maximum and apparent cutoff in the white dwarf LF derived from this sample has already been used to infer an age for the local galactic disk population (Winget et al. 1987), there are several ways in which the function is likely to be incomplete. Each of these 
is discussed below, and underscores our caution that the empirical LF is not necessarily accurate enough yet to infer many details about the star formation history of the disk population.

(a) Possible existence of stars much fainter than $M_{v} \sim+16$.

It is asserted in LDM that this sample and others discussed in that paper show that there is a sharp drop in the $L F$ at $M_{v}$ fainter than 16.5. Note that the preliminary trigonometric parallax reported for the southern proper motion star ER8 by M. T. Ruiz at this meeting indicates that this star also lies near this empirical luminosity limit. The space densities of stars at $M_{v} \sim 17-17.5$ must be reduced by more than an order of magnitude from the peak. However, there is very little search volume available from this sample to test the numbers of stars at $M_{v}>+18$, if such objects avoid low mass main sequence stellar companions. The constraints these results place on a bimodal star formation hypothesis (e.g., Larson 1986) are discussed in LDM.

\section{(b) Unidentified members of binary systems.}

Two kinds of unresolved binaries may hide white dwarfs from this census. First, there are those white dwarfs with bright nondegenerate companions (the "Sirius B" type). Insofar as close binaries tend to have mass ratios near unity, there could be a fair fraction of $A-K$ dwarfs, subgiants and giants with white dwarf companions. It is still controversial as to what fraction of G stars, for example, are binary; however, it is often impossible to distinguish a lower luminosity main sequence companion from a degenerate companion for single-lined spectroscopic binaries. For wide binaries the incidence of white dwarf-nondegenerate pairs appears to be rather small (Greenstein 1986). However, the frequency of binaries with an evolved component and their period distribution are certainly not well determined. In the solar neighborhood, the census of stars within $5.2 \mathrm{pc}$ of the Sun (cf. van de Kamp 1971), often regarded as a nearly complete sample, contains two single white dwarfs, two with distant, low luminosity nondegenerate companions, and two white dwarfs (Sirius B and Procyon B) with close, bright companions which could not be found by the usual techniques.

Secondly, there are now known to be unresolved binary white dwarfs, such as L870-2 (Saffer, Liebert and Olszewski 1988). The frequency of such objects is not well determined, especially for periods longer than several hours (see Robinson and Shafter 1987). However, the frequency of progenitor binaries with the required small separations is surely small enough that the effect of unresolved degenerate pairs is unlikely to exceed (conservatively) $10 \%$.

(c) Allowance for low velocity stars.

While the $1 / V_{\max }$ method as applied by Schmidt (1975) is designed to define a correct, effective search volume based on the limits of both apparent magnitude and proper motion, there may be significant numbers of white dwarfs missed which have small tangential velocities $\left(\mathrm{v}_{\tan }\right)$, despite the minimum ages (set by the cooling times) of at least $1 \mathrm{Gyr}$ and the well known increase in space motions with stellar age. This problem is clearly worse for the brighter stars 
near $M_{v} \sim+13$, which are found at greater distances and are also likely to have a younger mean age, than for the critical stars near the faint end used in defining the turnover of the LF.

It is instructive to consider the distribution of $v_{\tan }$ with $M_{v}$ for this sample (Figure 2). Here we also include stars in the LHS Eight Tenths Sample brighter than $M_{v}=+13$, although these were not included in the determination of the disk LF since the Palomar Green Survey is a much superior sample. While there hotter stars of low velocity, no cool stars are present with $\mathrm{v}_{\mathrm{tan}}<$ $40 \mathrm{~km} \mathrm{~s}^{-1}$. For an earlier sample (Liebert 1978), we compared the distribution of tangential velocities for the known cool white dwarfs near the Sun with those of a strictly color-selected sample of nearby $M$ dwarf stars - the Gliese/McCormick sample - and with the van de Kamp 5 pc sample of $\mathrm{K}-\mathrm{M}$ dwarfs. The comparison suggested that a correction factor of up to $100 \%$ for missing low velocity white dwarfs was possible, if the white dwarfs have the same mean age a.s the low mass main sequence stars. However, as each white dwarf with $M_{v}>+13$ has a minimum age of at least $1 \mathrm{Gyr}$, and a nuclear-burning lifetime before that, the mean age of this sample is likely to be significantly older. Moreover, if cool, low velocity white dwarfs were present in comparable numbers to those constituting the known sample, it seems improbable that not a single representative of this group with $v_{\tan }<40 \mathrm{~km} \mathrm{~s}^{-1}$ would have been found.

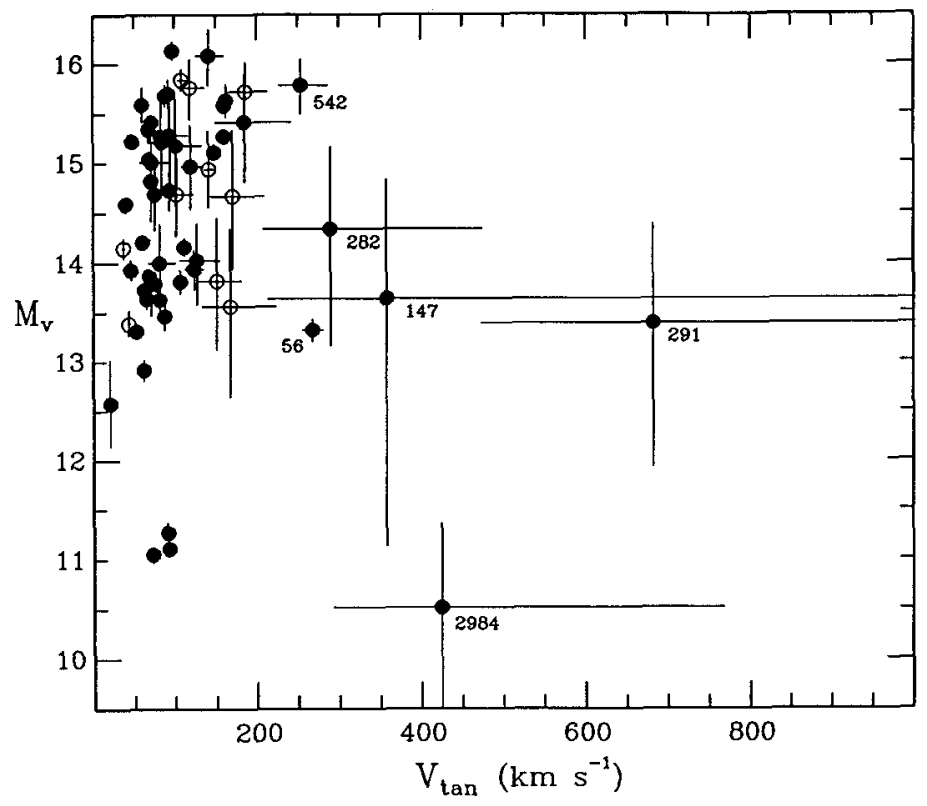

Figure 2. The distribution of tangential velocities $\left(v_{t a n}\right)$ against absolute visual magnitude $\left(M_{v}\right)$ with error bars shown. Filled circles are data points from this paper, for LHS stars with $\mu \geq 0$ ". 8 . Open circles are a new set of data presented in the following paper (Dahn et al. 1989), for LHS stars with $0 ! 700 \leq \mu \leq 0$ !'799 $\mathrm{yr}^{-1}$.

Finally, the nice matchup between these bins derived from the proper motion sample (open circles in Figure 1) with the kinematically-unbiased hot star sample (filled circles) and the model slope of Winget et al. (1987) suggests that the effect is not substantial even for the brighter intervals of the proper motion sample. Thus, it is unclear as to what correction needs to be 
applied for the missing low velocity stars to define better the shape of the empirical LF. It is clear that the omission of significant numbers of low velocity stars could distort the true shape.

(d) Possible scale height inflation.

Some authors have suggested (Iben and Tutukov 1984; Garcia-Berro et al. 1988) that substantial numbers of cool white dwarfs could be missing from the solar neighborhood due to a greatly increased scale height associated with their larger mean age than that of a typical sample of stars in the solar neighborhood. This effect could be especially important if carbon and oxygen separate in the core, releasing additional energy and slowing the cooling rate by 1.6 Gyr per $0.1 \mathrm{M}_{\odot}$ of deposited oxygen (Garcia-Berro et al. 1988). Later calculations (Barrat, Hansen and Mochkovitch 1988) indicate that the effect on the white dwarf cooling rate is much more modest, adding only $\sim 1 \mathrm{Gyr}$ to the cooling ages.

However, we have a potential empirical test as well: If there were a population of old, dispersed white dwarfs with a scale height 6.6 times that of the typical old disk (Garcia-Berro et al., F. 146), we may expect the sample in the solar neighborhood to reflect this in the mean motions, which should scale approximately as $\mathrm{v}_{\mathrm{tan}} \sim \mathrm{z}$ for a harmonic law. Thus, the mean tangential velocity should "inflate" by a factor of 6 between $M_{v}=+13$, a luminosity too high for oxygen settling, and $M_{v}=+15-16$. An examination of Figure 2 suggests a modest indication of an increasing $v_{t a n}$ between $M_{v} \sim+13$ and +16 , as expected for the increasing mean age of the sample without the inclusion of gravitational settling in the cooling curve. However, the difference in mean velocity among the magnitude bins is not statistically significant. There is little evidenca in this data set for the effect of scale height inflation amongst the coolest white dwarfs.

In summary then, it is difficult to quantify the various effects discussed above into a statement about how incomplete the empirical LF reported here might be. The effect of excluded binaries should not exceed the space density of luminous stars plus $10 \%$ of the white dwarfs themselves. We argued that the incompleteness due to low velocities is at most a factor of two for the coolest stars, but is likely to be much less. The effect of scale height inflation in pulling stars out of tha plane is also likely to be modest. The total mass density of white dwarfs in the solar neighborhood derived from this sample (using a mean mass of $0.6 \mathrm{M}_{\odot}$ ) is only $0.002 \mathrm{M}_{\odot} \mathrm{pc}^{-3}$. It is possible that this density could be as much as doubled by correcting for these effects.

\section{A PRELIMINARY LUMINOSITY FUNCTION FOR WHITE DWARFS OF THE LOCAL HALO}

Several of the white dwarfs in this sample clearly have high enough space velocities to be considered interlopers from the halo population. In fact Schmidt (1975) first applied the $1 / \mathrm{V}_{\max }$ method to estimating the local density of halo stars from a bright proper motion sample. This sample included only one white dwarf. He assumed (1) that such a population would have a mean $v_{\tan }=250 \mathrm{~km} \mathrm{~s}^{-1}$, based on Oort's (1965) studies of metal-poor RR Lyrae stars; (2) that all stars 
with velocities above this value were assignable to the halo population; but (3) that stars falling in the lower half of the distribution would be counted as disk population members. Thus, the density derived from the assigned stars should be corrected upwards by a factor of two.

Depending on the possible existence of a "thick disk" population (Gilmore and Reid 1983), or of an extended non-gaussian, high-velocity tail to the velocity distribution of old disk stars, the Schmidt (1975) assumptions may be too simplified. However, even for an assumed scale height of order $1 \mathrm{kpc}$, few hypothetical "thick disk" stars would be expected to have tangential velocities reaching $250 \mathrm{~km} \mathrm{~s}^{-1}$. For a first look at the luminosity function of local halo white dwarfs, let us simply adopt the same prescription, so that comparisons may be made with the total LF of visible halo stars derived by Schmidt in exactly the same way.

Six white dwarfs in this sample have $\mathrm{v}_{\tan }>250 \mathrm{~km} \mathrm{~s}^{-1}$ and are assignable to the halo sample; five of these are fainter than $M_{v}=+13$. None of the other stars have estimated tangential velocities exceeding $200 \mathrm{~km} \mathrm{~s}^{-1}$, although some error bars overlap with those of the assigned halo sample. In Figure 3, the LF derived from these six stars is displayed with the disk LF binned in unit $M_{v}$ intervals, applying the blackbody $\mathrm{BC}$ values to both disk and halo degenerate stars.

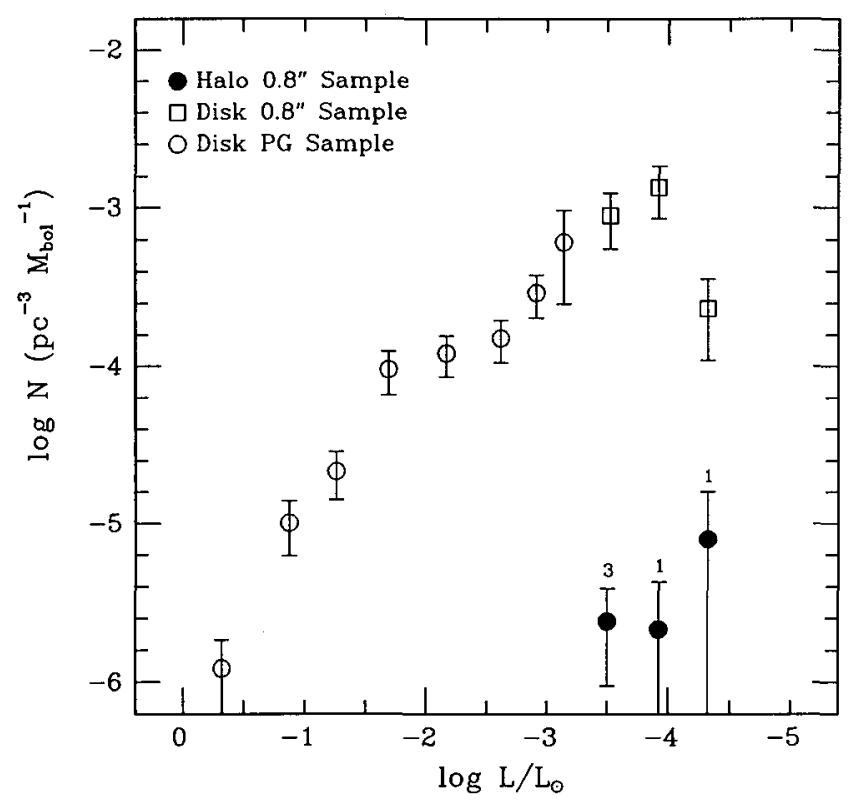

Figure 3. Luminosity functions for the local disk and halo, binned in one bolometric magnitude intervals with blackbody BCs applied.

The total space density of the designated halo sample is $1.3( \pm 0.6) \times 10^{-5} \mathrm{pc}^{-3}$, with poisson error bars. Let us compare this with the subset of Schmidt's (1975) Table 2 with $\mathrm{v}_{\text {tan }}>250 \mathrm{~km}$ $\mathrm{s}^{-1}$. These add up to total number densities of nondegenerate and degenerate stars of $5.75 \times 10^{-5}$ and $3.69( \pm 3.69) \times 10^{-5} \mathrm{pc}^{-3}$, respectively. The former was derived from 17 main sequence stars with assigned masses between 0.25 and $0.75 \mathrm{M}_{\odot}$ for which Schmidt assumed the LF was reasonably complete, except for the correction for the low velocity part of the distribution. The latter number was based on one white dwarf star, so that our new result should be an improvement. 
If the local halo LF may be approximated as a Salpeter (1955) power law, a comparison of the white dwarf density with that for nondegenerate stars can give a measure of the slope. We shall again follow Schmidt in assuming that the latter applies to the main sequence only in mass range $0.25-0.75 \mathrm{M}_{\odot}$. The white dwarf density should include stars above the appropriate halo turnoff mass of $\sim 0.8-0.9 \mathrm{M}_{\odot}$. Since we can make no claims to be complete for white dwarfs as faint as $M_{v} \sim+15$, this white dwarf LF may not provide a complete census for masses up to the mass limit for the production of neutron stars. The exercise thus yields an upper limit to the power law slope for the local halo mass function.

The ratio of the number density of degenerate stars from this work to nondegenerate stars, as defined above from Schmidt (1975) is $0.22 \pm 0.11$. For Salpeter mass distributions of the form

$$
\xi(\mathrm{M}) \sim \mathrm{M}^{-\alpha}
$$

the number ratios of stars for the $0.8-60 \mathrm{M}_{\odot}$ and $0.25-0.75 \mathrm{M}_{\odot}$ mass intervals are 0.12 for $\alpha=$ 2 and 0.49 for $\alpha=1$, respectively. Our white dwarf space density thus indicates that the local halo mass function slope is not steeper than 2 over this wide range of mass, and may well be considerably flatter, as inferred from proper motion data by Reid (1984). We note that there are contrasting claims for power law slopes in deep CCD analyses of the main sequences of various globular clusters.

The Schmidt prescription leads to a total space density of local halo white dwarfs of 2.6 ( \pm $1.2) \times 10-5 \mathrm{pc}^{-3}$. This result is two orders of magnitude lower than that derived previously for the white dwarfs of the disk population, before any corresponding correction for the missed low velocity stars. Neither number includes a correction for missed binaries. This ratio for the dying stars compares with ratios derived for main sequence stars of $1 / 253$ from Hartwick, Cowley and Mould (1984), 1/600 by Dawson (1984), or $1 / 200$ by Chiu (1980). The degenerate star ratio, if complete, ought to be somewhat lower since a larger effective interval of the mass function has formed white dwarfs in the halo population.

This first sampling indicates that more extended proper motion samples complete for higher proper motions and to fainter absolute magnitudes may achieve a meaningful description of the local LF for halo white dwarfs. It is curious that one halo star already shows up in the somewhatsparse faintest magnitude bin, giving that interval a correspondingly high (but precarious) space density. We permit ourselves two premature remarks: First, the halo LF may obviously form a peak at lower luminosity if this is an older population than the local disk. Secondly, the theoretical LF calculations of Iben and Laughlin (1988) assuming a delta function or "impulse" star formation $10^{10}$ years ago lead us to anticipate a steeper slope prior to the peak in the halo LF.

We thank Matt Wood for pointing out the discrepancy in the hot star luminosity scale discussed in Section 2 here. This work was supported by the National Science Foundation through grant AST 88-40482. 


\section{REFERENCES}

D'Antona, F. and Mazzitelli, I. 1986, Astron. Astrophys., $162,80$.

Barrat, J. L., Hansen, J. P. and Mochkovitch, R. 1988, Astron. Astrophys., 199, L15.

Chiu, L.-T. G. 1980, Astron. J., 85, 812.

Dahn, C. C., Monet, D. G. and Harris, H. C. 1989, following paper in these proceedings

Dawson, P.C. 1986, Astrophys. J., 311, 984.

Garcia-Berro, E., Hernanz, M., Mochkovitch, R. and Isern, J. 1988, Astron. Astrophys., 193, 141.

Gilmore, G. and Reid, N. 1983, Mon.Not.R.A.S., 202, 1025.

Greenstein, J. L. 1986, Astron. J., 92, 859.

Hartwick, F. D. A., Cowley, A. P. and Mould, J. R. 1984, Astrophys.J., 286, 269.

Iben, Jr., I. and Tutukov, A. V. 1984, Astrophys. J., $282,615$.

Kovetz, A. and Shaviv, G. 1976, Astron. Astrophys., 52, 403.

Larson, R. B. 1986, Mon.Not.R.A.S., 218, 409.

Liebert, J. 1978, Astron. Astrophys., 70, 125.

Liebert, J. 1980, Ann. Rev. Astr. Ap., 18, 363.

Liebert, J., Dahn, C. C., Gresham, M. and Strittmatter, P. A. 1979, Astrophys. J., 233, 226.

Liebert, J., Dahn, C. C. and Monet, D. G. 1988, Astrophys. J., 332, 891.

Luyten, W. J. 1979, LHS Catalogue, (Univ. of Minnesota: Minneapolis), 2nd Ed.

Oort, J.H. 1965, in Galactic Structure, ed. A. Blaauw and M. Schmidt, (Univ. of Chicago: Chicago), p. 455.

Reid, N. 1984, Mon.Not.R.A.S., 206, 1.

Robinson, E. L. and Shafter, A. W. 1987, Astrophys. J., 322, 296.

Saffer, R. A., Liebert, J. and Olszewski, E. 1988, Astrophys. J., 334, in press.

Schmidt, M. 1975, Astrophys. J., 202, 22.

Sion, E.M. and Liebert, J. 1977, Astrophys. J., 213, 468.

van de Kamp, P. 1971, Ann. Rev. Astr. Ap., 9, 103.

Weidemann, V. 1967, Zs. f. Astrophys., 67, 286.

Winget, D.E., Hansen, C.J., Liebert, J., Van Horn, H.M., Fontaine, G., Nather, R.E., Keplei', S.O. and Lamb, D.Q. 1987, Astrophys.J. (Letters), 315, L77.

Winget, D.E. and Van Horn, H.M. 1987 in The Second Conference on Faint Blue Stars, IAU Coll. 95, eds. A.G.D. Philip, D.S. Hayes and J. Liebert, (L. Davis Press: Schenectady NY) p. 363. 